\title{
Measuring handicap: motives, methods, and a model
}

\author{
Rowan H Harwood, Sutthichai Jitapunkul, Edward Dickinson, Shah Ebrahim
}

Medicine is concerned with the manifestations and consequences of disease, but patients' views on how ill health affects them may differ from those of their doctors. ${ }^{12}$ The increasing importance of chronic diseases, and the need to accommodate patients' views, have prompted a reassessment of the way we consider the effects of disease. Most patients now seen in primary care and hospital have chronic, irreversible diseases that do not always lead to immediate or inevitable death but cause disability and disadvantage. ${ }^{3}$ Modern medicine has developed excellent technologies to measure the immediate consequences of disease, but our ability to appreciate and quantify the wider aspects is less advanced. ${ }^{4}$ Such measures are needed if we are to be able to assess the outcomes of health care.

\section{A framework for measuring health}

The International Classification of Impairments, Disabilities and Handicaps (ICIDH $)^{5}$ has attempted to clarify the consequences of chronic disease by offering a new taxonomy. An impairment is any loss or abnormality of psychological, physiological, or anatomical structure or function (for example, shortness of breath or weakness in a limb). A disability is any restriction or lack of ability to perform a task or activity (such as walking, dressing or maintaining continence). Handicap is the disadvantage suffered by an individual as a result of ill health, due to the inability to fulfil a role which is normal for someone of that age, sex, and culture. ${ }^{5}$ Doctors are used to defining impairments as part of history taking and examination, but disabilities and handicaps are often of greater concern to patients.

\section{Why measure handicap?}

The main motive for measuring handicap is to use it as an outcome measure. This is required for trials of new therapies in chronic disease (drugs, operations, physiotherapy techniques, specialist rehabilitation units), for studies of cost effectiveness, and for evaluating clinical services (for audit, for assessing service innovations, or in monitoring contracts for health care provision, where a quantitative outcome assessment is required).

Defining impairments and disabilities is useful in diagnosis and understanding how ill health has its impact on someone's life. An accurate diagnosis may allow a specific therapy to be implemented. Measuring impairments allows the natural history of an illness or response to therapy to be assessed (such as limb weakness after a stroke or peak expiratory flow rate in obstructive airways disease). When there is no specific therapy, assessing disability can lead to rehabilitative interventions such as teaching someone with a hemiplegia to dress or manoeuvre a wheelchair. However, impair-

. . impairments and disabilities
are incomplete and inadequate
outcome measures in chronic
disease.

ments and disabilities are incomplete and inadequate outcome measures in chronic disease. Some apparently trivial impairments may result in major handicap (for example, a facial scar may lead to intolerable feelings of inadequacy, depression, and social isolation). Despite considerable work on devising and validating disability scales, ${ }^{6-10}$ their use in clinical trials has sometimes failed to show improvement where clinical or other evidence suggested that benefits should be found. ${ }^{11-13}$ Assessments of impairment, disability, and handicap may be conflicting; in heart failure there are poor correlations between measurements of cardiac output (an impairment), treadmill exercise tolerance and timed walking tests (disabilities), and customary mobility measured by pedometer (an aspect of handicap). ${ }^{14}$ The lack of a direct relation between impairment, disability, and handicap also applies in chronic airways disease..$^{15} 16$ Finally, from the patient's point of view, impairments and even disabilities matter less than their effect on everyday life.

Some outcome measures have tried to embrace wider concerns. Measures of perceived health or "quality of life" have been used with some success ${ }^{17-19}$; examples include the Nottingham health profile, sickness impact profile, quality of wellbeing scale, and McMaster health index..$^{20}$ Defining "quality of life" and "health" precisely is difficult, but these scales generally contain items about symptoms, feelings, attitudes, impairments, disabilities, and handicaps. For example, the sickness impact profile has 136 items in 12 categories including eating, ambulation, recreation, and social interaction. The Nottingham health profile has 38 items in six dimensions (mobility, pain, emotion, social isolation, sleep, and energy) in part 1, and asks about problems in seven areas of daily life in 
part 2. The quality of wellbeing scale has dimensions of mobility, physical activity, and social activity and a checklist of symptoms. Sensitivity to change as a result of interventions will depend on the extent to which the items measured are relevant to the type of intervention (and hence how many of the items are relevant). This has been shown to be poor in some cases. ${ }^{21}$

Quality of life is influenced by many factors other than health - for example, relationships, wealth, interests, and employment. A study of the impact of a health promotion and exercise package for older people failed to demonstrate changes measured by the Nottingham health profile, but mean scores were twice normative values, reflecting the inner city residence of the sample studied. ${ }^{22}$ Probably any quality of life benefits from the health promotion programme were trivial compared with the adversity associated with socioeconomic deprivation.

A further problem of some multidimensional quality of life indicators is that associated with aggregation of the data into a single index. When using the Nottingham health profile results from each of six dimensions and seven questions have to be considered separately, and no means of pooling the information has been developed; thus the interpretation of inconsistent findings between dimensions is impossible. The sickness impact profile generates an overall score simply by adding the scores of individual dimensions. The quality of wellbeing scale is more advanced in weighting responses on the three dimensions and the symptom list before adding them.

Although alleviating some impairments (pain, nausea, anxiety) or disabilities (incontinence) may be a valid objective of healthcare interventions in its own right, impairment and disabilities will often have handicaps associated with them, such as limited mobility or physical independence due to pain or restriction of occupation and social integration due to anxiety or incontinence. The reversal of impairments and the reduction of disability are important strategies to explore in rehabilitating people with chronic disease. However, many diseases (for example, stroke, dementia, and arthritis) are associated with irreversible impairments and disabilities. Measuring reduction in disability (strictly defined in terms of the ability to perform tasks or activities) takes no account of what someone actually does in their everyday life. If someone cannot walk they may benefit from being taught how to use a wheelchair. However, the ability to use a wheelchair is irrelevant if it isn't actually used. This will depend on factors like motivation (does the subject want to use it?), physical environment (unadapted steps or stairs?), relationships (is there someone to push?), and resources (an electric-powered wheelchair, an adapted car or flat). Some abilities may be unimportant or irrelevant to a given individual. The Barthel index ${ }^{\circ}$ includes items on climbing stairs and bathing, but climbing stairs may be of no concern to someone who lives in a house without stairs and who has no desire to go anywhere which has and the inability to use the bath independently may be of little additional consequence to someone who also needs washing and dressing. Rehabilitation has not necessarily failed where disabilities are irremediable. Reducing handicap covers a wide range of rehabilitative interventions, including resettlement in appropriate accommodation; providing services such as home nursing, domestic help, and day centres; and psychological adjustment to illness. Reducing handicap must be the criterion by which intervention at any level is judged. As with quality of life scales, measures that fail to separate out the different consequences of ill health lose precision as some items will be redundant or inappropriate.

Current methods of measuring handicap As described, the relation between impairment, disability, and handicap is not straightforward. ${ }^{5}{ }^{15}$ Similar degrees of impairment can lead to different disabilities and similar disability produces different levels of handicap. $=1+1023$ Thus we cannot infer levels of handicap from information on impairments or disabilities.

\section{.. we cannot infer levels of handicap from information on impairments or disabilities.}

The ICIDH is a classification, not a measurement scale. ${ }^{2+}$ Its handicap section defines six basic "survival roles," which describe disadvantage in orientation (the ability to perceive and understand the immediate environment including sight, hearing, and cognition), physical independence (from human or mechanical assistance), mobility (the distance one can move from one's bed), occupation (employment, domestic work, and recreation), social integration, and economic self sufficiency (including the ability to earn an income and the possession of resources enabling problems to be overcome). These survival roles may be thought of as dimensions into which more complex roles (such as professional, parent, or citizen) can be resolved. Handicap is classified according to the disadvantage associated with deficiencies in each dimension. An example of different levels of disadvantage is evident for the dimension mobility: bedfast or chairfast, roombound, housebound, confined to immediate neighbourhood, unlimited mobility. The emphasis is not on how you get there but on how far you get in practice.

We identified four putative handicap measures: the modified Rankin scale, ${ }^{25}{ }^{26}$ the Jefferys measure, ${ }^{27}$ the functional autonomy measurement system (système de mesure de l'autonome functionelle (SMAF),,$^{28}$ and the Edinburgh rehabilitation status scale. ${ }^{29}$ None 
of these instruments proved entirely suitable for measuring handicap. The Jefferys scale, ${ }^{27}$ used in the 1971 Office of Population, Censuses, and Surveys handicap survey ${ }^{30}$ and in community surveys in Canterbury, ${ }^{23}$ measures disability. The other three use ICIDH definitions and attempt to measure global handicap. The SMAF estimates handicap by identifying disabilities which, are not compensated for by aids or assistance. This is an attractive approach but remains limited by some of the criticisms of disability scales. Moreover, compensating disabilities is only one of the ways in which disadvantage can be reduced. Provision of health service resources tends to be concentrated on those people with moderate or severe physical disability, but the impact of these resources in alleviating disability (as opposed to handicap) may be very limited. Availability, acceptability, and accessibility of services are also important. Therefore, this instrument may not be suitable for comparing populations which have dissimilar service systems, such as differing emphases on institutional and domiciliary care. Although the "modified Rankin scale"26 was derived from the original Rankin disability scale $^{25}$ by introducing the phrases "interferes with life style" or "restriction of life style" in some grades, other descriptions are still of disability rather than handicap. It covers only the physical independence dimension of handicap in the ICIDH classification, and with only six points, while simple, is relatively crude. The Edinburgh rehabilitation status scale describes itself as a "measure of medicosocial dysfunction", and has dimensions covering dependency, activity, social integration, and "the effects of symptoms on lifestyles". ${ }^{29}$ Activity and social integration closely reflect ICIDH roles, but the others are more global. No attempt was made to weight items on the scale; the scaling is thus arbitrary. Nevertheless, some evidence was provided that it was sensitive to changes occurring during rehabilitation.

Another group of measures seek to assess handicap due to specific impairments or disabilities. Assessment of auditory handicap is most advanced, with several valid and reliable measures [for example reference 31]. All include items that would be classified as disability under the ICIDH. This criticism can also be levelled at specific handicap measures relating to dental health ${ }^{32}$ and the impact of dizziness. ${ }^{33}$ Some other measures allude to handicap, such as the New York Heart Association classification ${ }^{34}$ and the Rosser and Kind quality of life index disability subscale. ${ }^{35}$ Both of these are short and rather limited in scope.

\section{A new model for measuring handicap}

To quantify handicap three questions must be addressed, as follows ${ }^{36}$ :

- How are states of health to be described?

- How are different health states to be valued relative to each other?

- How are individuals' values to be transformed into a group value?
The system for describing different states of health needs to be comprehensive enough to cover all possible states incurring disadvantage, sensitive enough to allow meaningful changes to be measured, yet clearly enough worded to achieve acceptable reliability. The use of the "survival roles" described in the ICIDH as different dimensions to describe health states seems particularly appropriate. By categorising disadvantage on each of these dimensions an individual subject's handicap can be described.

To determine how different health states should be valued, and how results should be aggregated, we must examine the origins of handicap. An individual's role in life is influenced by many factors other than abilities or disablities, including social, cultural, economic, and psychological factors. Each individual presumably balances these factors to reduce perceived disadvantage and achieve personal satisfaction. A change in any factor should prompt a new equilibrium point which optimises satisfaction. Those who are affected by impairment or disability will adopt a role that maximises satisfaction with life within their limitations. When the adopted role is disadvantageous compared with the norm for someone of the same age, sex, and background, the individual is classified as handicapped. Pursuit of satisfaction is the motivation behind the choice of different roles, and dissatisfaction is an expression of perceived disadvantage and role preference, which are highly subjective features. Two people suffering from the same disability may differ in the disadvantage experienced because of differences in expectations and ambitions and in the extent to which the disability interferes with their normal activity. For instance, a woman with impaired hands due to rheumatoid arthritis cannot dress her baby which affects her maternal role. Another woman with the same disability is more concerned about her inability to drive which interferes with her social life and work opportunities. A wealthy business woman in the same position may have unrestricted work, travel and family capabilities by virtue of the nature of her work and the resources she can use to overcome problems.

Measuring handicap directly ...
is difficult since the reference
standard is normality ....

Measuring handicap directly in a given individual is difficult since the reference standard is "normality", and this covers a wide range of abilities, achievements, and expectations. Moreover, physical environment, resources and social situations, all of which impinge on handicap, are very diverse. All we can do is describe qualitatively an individual's unique predicament and assess their satisfaction with that state of affairs. To develop a quantitative measure from this we 
can use the econometric concept of "utilities" to measure the severity of handicapped states. The utility of a health state is the "value" or "worth" people put on it. ${ }^{37}$ By convention, a fully healthy state is given a utility of $+1 \cdot 0$, and death a utility of $0 \cdot 0$ (states "worse than death" can have negative values). Utilities represent non-arbitrary values reflecting the relative desirability of various states, at an interval level of measurement. Clearly, utilities will reflect relative preferences and values, and will be closely related to satisfaction.

We could measure utilities by asking individuals to rate the desirability of their own state of health against an idealised normal state. In practice this is very difficult, although it has been described as a measure of stress in carers. ${ }^{38}$ For many epidemiological and health services research purposes we need to measure a variable in a population or group rather than knowing individual values per se. In these cases we can describe each individual's health (by classifying each to the appropriate level on each of the six handicap dimensions), then apply a valuation of that description that has been predetermined from a study of a representative group of "judges". The use of utilities averaged from representative judges means that an individual's handicap score will represent his or her handicap only to the extent that his or her views coincide with those of the judges. However, it does allow measurements to be made on important groups whose subjective opinions of their own health would be difficult to obtain, such as those with communication disabilities or cognitive impairment (since we can describe their state of health objectively and then apply the valuations of those states made by the judges). The assumption has to be made that valuations made by judges able to take part in a valuation exercise would be the same as patients' who are not. The same arguments apply equally to other health indices with scale weights derived from groups of judges, including the sickness impact profile, Nottingham health profile, quality of wellbeing scale, and Rosser-Kind index. There have been some interesting recent attempts to individualise the items and scale weights in quality of life measures (D Ruta et al, third European Health Services Research Conference, London, 1991), ${ }^{39}$ but these have had to limit themselves to a few items and require considerable cooperation and cognitive ability from subjects.

\section{A market research solution}

A comprehensive, quantitative scale requires a valuation of the severity of all the possible states described by the health state descriptive system. In the ICIDH $^{j}$ each of the six dimensions was given nine levels of severity (from no disadvantage to extreme disadvantage). Any state of health can be described by a combinations of one level from each of these six dimensions, giving 531441 $(9 \times 9 \times 9 \times 9 \times 9 \times 9)$ possible combinations (even though some of them are improbable; such as being severely disadvantaged in orientation and mobility, yet normal in physical independence). To measure the utility of each potential state one at a time would clearly not be feasible. However, if the dimensions are relatively independent (or at least can be imagined as being independent), various statistical techniques can be used to construct mathematical models for estimating utilities for any state from measurements made on a small sample of states. For example, Torrance et al used "multi-attribute utility theory" in developing a general health index. " However, this method makes a number of arguable assumptions and is computationally difficult. A simpler alternative called conjoint analysis has been developed for use in market research. ${ }^{11}$ :

Market researchers commonly face a problem exactly analogous to that described here. They need to know which characteristics of a product or service are most important to consumers. An ideal product will have all the best characteristics, but in practice trade offs are required - for instance, between the performance, reliability, fuel consumption, and price of a car. Each of these characteristics will have several possible "levels", such as a high, medium, or low fuel consumption. Product designers need to know the effect that each level of each different characteristic has on overall desirability. The problem is that if there are several different characteristics, and each has several possibilities, the total number of possible combinations soon becomes unmanageable (as with our descriptions of handicap). Conjoint analysis depends on the ability of subjects to make judgements about hypothetical products or states of health. Series of states are evaluated, each being described by a combination of different levels of each characteristic (in this case different severities of disadvantage in mobility, physical independence, occupation, and so on). Respondents may be unable to explain which characteristics they are using in making a valuation or how they are combining them to form overall judgements. However, conjoint analysis infers a value system from these judgements. The term "conjoint" refers to this process of joint evaluation. In developing a handicap measurement scale we wish to know the relative contributions of each of the severities within the six dimensions of handicap.

The technique for modelling responses is computer-dependent and iterative. ${ }^{13}$ Given a set of judgements about health states, the program assigns arbitrary "part utilities" to each level of each characteristic. These part utilities are used to calculate an estimated overall utility for each health state (usually using an additive model and ignoring interactions), the estimates are compared with the measured values and a goodness of fit statistic is calculated. The part utilities are then systematically varied until the calculated values correspond as closely as possible to the data. Fortunately the procedure does not require data from all the possible combinations of different levels of each 
characteristic; estimates can be made from a small sample if the states are varied in certain systematic ways. Although interactions are theoretically likely (in market research, most improvements to a product affect its price, and physical independence is partly dependent on mobility), as a general rule the simplest possible model is chosen, and its robustness then tested in practice (as part of validation).

A method must be chosen for determining the utility of health states to provide the raw data for the modelling process. Utilities can be measured by a number of techniques, including the standard gamble, time trade off, or simpler magnitude estimation or visual analogue scale. Some work suggests that they give approximately similar results, ${ }^{44}$ although other evidence is contradictory. There is no clear advantage for any one method on theoretical grounds. Often simple ranking of severity (or desirability) is used.

With the approach described here, a scale can be constructed comprising a handicap classification questionnaire (enabling an individual's health state to be described in terms of different levels of disadvantage on each of the six dimensions), and a matrix of scale weights relating to each level in each dimension (the "part-utilities" from the conjoint analysis). A formula is then used to combine them into a final handicap score, representing an estimate of the utility that the "judges" would have given that state were they to have rated it directly. An accompanying paper in this issue ( $p 11)^{45}$ describes such a scale we developed from these ideas.

SJ was supported by the British Council and ED by the Roya College of Physicians of London; RHH is an MRC health services research training fellow.

1 Bury MR. The ICIDH: a review of research and prospects. International Disability Studies 1987;9:118-28.

2 Williams GH. Disablement and the social context of daily activity. International Disability Studies 1987;9:97-102.

Royal College of Physicians. Physical disability in 1986 and beyond. $\mathcal{F} R$ Coll Physicians Lond 1986;20:160-94.

4 Seale C, Davies P. Outcome measurement in stroke rehabilitation research. Int Disabil Studies 1987;9: 155-60.

5 World Health Organisation. International Classification of Impairments, Disabilities and Handicaps. Geneva: WHO, 1980

6 Mahoney FI, Barthel DW. Functional evaluation: the Barthel index. Maryland State Medical foumal 1965; 14:61-5.

7 Nouri FM, Lincoln NB. An extended activities of daily living scale for stroke patients. Clinical Rehabilitation living scale for

8 Martin J, Meltzer H, Elliot D. The prevalence of disability among adudlts. London: HMSO, 1988. (OPCS Socia Survey Division.)

9 Katz S, Ford AB, Moskowitz RW, Jackson BA, Jaffe MW. Studies of illness in the aged. The Index of ADL: standardised measure of biological and psychosocial function. $\mathcal{F} A M A$ 1963;185:914-9.

10 Ebrahim S, Nouri F, Barer D. Measuring disability after a stroke. 7 Epidemiol Community Health 1985;39:86-9.

11 Bond J, Gregson BA, Atkinson A. Measurement of outcomes within a multi-centred randomised controlled trial in the evaluation of the experimental NHS nursing homes. Age Ageing 1989;18:292-302.

12 Gilchrist WJ, Newman RJ, Hamblen DL, Williams BO. Prospective randomised trial of an orthopaedic geriatric inpatient service. BMF 1988;297:1116-8.

13 Garraway WM, Akhtar AJ, Hockey L, Prescott RJ. Management of acute stroke in the elderly: follow up of a controlled trial. BMF 1980;281:827-9.
14 Cowley AJ, Fullwood LJ, Muller AF, Stainer K, Skene AM, Hampton JR. Exercise capability in heart failure: is cardiac output important after all? Lancet 1991;337: $771-3$.

15 Ebrahim S. Measurement of impairment, disability and handicap. In: Hopkins A, Costain D, eds. Measuring the outcomes of medical care. London: Royal College of Physicians of London, 1990:27-41.

16 Williams SJ, Bury MR. "Breathtaking": the consequences of chronic respiratory disorder. International Disability Studies 1989;11:114-20

17 Caine N, Harrison SCW, Sharples LD, Wallwork J. Prospective study of quality of life before and after coronary artery bypass grafting. BMf 1991;302:511-6.

18 Croog SH, Levine S, Testa MA, Brown B, Bulpitt CJ Jenkins CD, et al. The effects of antihypertensive therapy on qualify of life. $N$ Engl f Med 1986;314:1657-64.

19 Bombardier C, Ware J, Russell IJ, Larson M, Chalmer A Read JL. Auranofin therapy and quality of life in patients with rheumatoid arthritis. Am $7 \mathrm{Med} 1986 ; 81: 565-78$.

20 Walker SR, Rosser RM. Quality of life: assessment and application. Lancaster: MTP Press, 1988

21 Fitzpatrick R, Ziebland S, Jenkinson C, Mowat A, Mowat A. Importance of sensitivity to change as a criterion for selecting health status measures. Quality in Health Care 1992;1:89-93.

22 Ebrahim S, Williams J. Assessing the effects of a health promotion programme for elderly people. 7 Public Healt Med 1992;14:199-205.

23 Warren MD. The Canterbury studies of disablement in the community: prevalence, needs, and attitudes. Int $\mathcal{f}$ Rehabil Res 1985;8:3-18.

24 Wood PHN. Maladies imaginaires: some common misconceptions about the ICIDH. International Disability Studies 1987;9:125-8.

25 Rankin J. Cerebral vascular accidents in patients over the age of 60. 2. Prognosis. Scot Med f 1957;2:200-15.

26 UK-TIA Study Group. The UK-TIA aspirin trial: interim results. $B M \mathcal{F}$ 1988;296:316-20.

27 Jefferys $M$, Hyman $M$, Millard JB. A set of tests for measuring motor impairment in prevalence studies. Fournal of Chronic Diseases 1969;22:303-19.

28 Hebert R, Carrier R, Bilodeau A. The functional autonomy measurement system (SMAF): description and validation of an instrument for the measurement of handicaps. Age Ageing 1988;17:293-302.

29 Affleck JW, Aitken RCB, Hunter JAA, McGuire RJ, Roy CW. Rehabilitation Status: a measure of medicosocial dysfunction. Lancet 1988;i:230-3.

30 Harris AI. Handicapped and impaired in Great Britain. London: HMSO, 1971

31 Schow RL, Nerbonne MA. Hearing handicap and Denver scales: applications, categories, interpretation. $\mathcal{F} A M A$ 1980;13:66-77.

32 Fiske J, Gelbier S, Watson RM. The benefit of dental care to an elderly population assessed using a sociodental measure of oral handicap. Br Dent f 1990;168:153-6.

33 Jacobson GP, Newman CW. The development of the Dizziness Handicap Inventory. Arch Otolaryngol Head Neck Surg 1990;116:424-7.

34 O'Brien B. Assessment of treatment in heart disease. In Teeling-Smith G, ed. Measuring health: a practical approach. Chichester: Wiley, 1988:191-210

35 Rosser RM, Kind P. A scale of valuations of states of illness: is there a social consensus? Int $\mathcal{f}$ Epidemiol $978 ; 7: 347-58$

36 Buxton M. Are we satisfied with QALYs? What are the conceptual and empirical uncertainties and what must we do to make them more generally useful? In: Hopkins A ed. Measures of the quality of life and uses to which such measures may be put. London: Royal College of Physicians, 1992:41-51

37 Von Neuman J, Morgenstern D. Theory of games and economic behaviour. 3rd ed. New York: Wiley, 1953.

38 Mohide A, Torrance GW, Streiner DL, Pringle DM Gilbert R. Measuring the well-being of family caregivers using the time trade-off technique. $f$ Clin Epidemiol using the time

39 O'Boyle CA, McGee H, Hickey A, O'Malley K, Joyce CRB. Individual quality of life in patients undergoing hip replacement. Lancet 1992;339:1088-91.

40 Torrance GW, Boyle MH, Horwood SP. Application of multi-attribute utility theory to measure social preferences for health status. Operational Research 1982;30:1043-69.

41 Churchill GA. Marketing research: methodological foundations. 3rd ed. Chicago: Dryden Press, 1989.

42 SPSS. SPSS categories. Chicago: SPSS, 1990.

43 Kruskal JB. Analysis of factorial experiments by estimating monotone transformations of the data. Fournal of the monotone transformations of the data. Fourmal
Royal Statistical Society Series B 1965;27:251-63.

44 Patrick DL, Bush JW, Chen MM. Methods for measurin levels of well-being for a health status index. Health Services Research 1973;8:228-45.

45 Harwood RH, Rogers A, Dickinson E, Ebrahim S. Measuring handicap: the London handicap scale, a new outcome measure for chronic disease. Quality in Health Care 1994;3:11-6. 